\title{
Accuracy of CBCT as modality to identify the presence of secondary mesiobuccal root canal in the maxillary first molar
}

\author{
Haikal Halil*, Mazlifa Mahidin*, Nik Nur Farahiyah*, Khairul Bariah Chi Adam** \\ *Department of Restorative Dentistry Kulliyyah of Dentistry International Islamic University \\ Malaysia, Malaysia \\ **Department of Oral Maxillofacial Surgery and Oral Diagnosis Kulliyyah of Dentistry International \\ Islamic University Malaysia, Malaysia
}

\begin{abstract}
Introduction: Successful endodontic treatment requires the clinician to be able to locate, disinfect, and obturate all canals presence in the root canal system to remove the infection and prevent re-infection. However, some canals, such as secondary mesiobuccal (MB) root canal often missed upon examination and the treatment. The success of locating these canals is determined by the methods used, a periapical radiograph and Cone Beam Computed Tomography (CBCT). The purpose of this study was to examine the sensitivity and accuracy of the periapical radiograph (PA) and CBCT on determining the presence of the secondary MB root canal. Methods: As much as 40 intact crown and intact radicular of the maxillary first molars, without root caries, root restoration, and endodontic treated, were taken as the samples. The presence of a secondary MB root canal was evaluated by a PA radiograph, CBCT, and clinical sectioning. Samples were undergone each test and sectioned after being completed the radiographic evaluation steps. Results: CBCT radiograph was successfully identifying $62.5 \%$ secondary MB root canal presence, whilst the PA radiograph has detected only $20 \%$ of the samples. The sensitivity of CBCT and PA radiograph was compared with the gold standard method, resulting 86.2 and $27.6 \%$ respectively. The statistical analysis showed that there was no significant difference between $C B C T$ test and the gold standard $(p=0.00)$. Conclusion: $C B C T$ was proven to be a reliable method to detect the presence of secondary $M B$ root canals due to its accuracy as high as the clinical sectioning compared to the PA radiograph.
\end{abstract}

Keywords: Cone Beam Computed Tomography (CBCT), periapical (PA) radiograph, secondary mesiobuccal $(M B)$ root canal, maxillary first molar, endodontic treatment 


\section{INTRODUCTION}

Endodontic treatment requires the clinicians on having a thorough knowledge of the root canal system. Hence, the success in endodontic treatment relies on the ability to locate, disinfect, and obturate all the root canal available in the root canal system. The endodontic procedure which needs the ability to locate all canals has been facilitated by the use of radiographic imaging. Hence it has been used as the major component in the management of the endodontic problems. ${ }^{1}$ Besides that, the periapical (PA) radiograph only reveals a two-dimensional image that results in superimposition of the teeth and surrounding tissues. Fortunately, this problem can be overcome by using the cone-beam computed tomography (CBCT) radiograph that produces images in three-dimensional view.

CBCT is a cone-shaped source of ionizing radiation that creates images in sections and reproduced it into a three-dimensional image. Research done by Scarfe et al. ${ }^{2}$ in 2007 stated that CBCT works by a single rotation of $180^{\circ}$ that during each exposure, hundreds of planar projections are incorporated to the required field of view (FOV). The advantage of the FOV is the improvement of the diagnostic accuracy especially in the evaluation of the accessory canals, calcified canal or missing canals. Besides that, the most significant advantage of CBCT is that it produces the image in 3-D view. Apart from radiographic evaluation, the tooth morphology itself also plays a role in determining the success of endodontic treatment. The variations of the amount of roots canal and the morphology are among the important factors will also be looked up so that the clinicians will not miss any root canals during treatment. Due to the complexity of the pulp spaces in root canal system, many studies have been conducted to help in identifying the morphology of complex root canal, includesing the complexity of secondary MB root canal system in maxillary first molars. Hence, it is appropriate to use the $\mathrm{CBCT}$ as the subject to study the complexity of the second $M B$ root canal in maxillary first molars. These can be correlated to the research conducted by Somma et al. ${ }^{3}$ in 2008 that stated that the incidence of people having secondary MB root canal is as much as $80 \%$ with a variation of fused $(58 \%)$ or independents
(42\%) second canal corresponds to the first canal. ${ }^{3}$ Another study done by Blattner et al. ${ }^{4}$ in 2010 has found that as much as $57.9 \%$ of secondary $M B$ root canal was presented when using $\mathrm{CBCT}$, while the gold standard was $68.4 \%$. This result proved that the CBCT is nearly as sensitive as the gold standard in detecting the presence or absence of secondary MB root canal. The purpose of this study was to examine the sensitivity and accuracy of the periapical radiograph (PA) and $C B C T$ on determining the presence of the secondary $M B$ root canal.

\section{METHODS}

For as much as 40 samples of extracted upper maxillary first molars were collected from several dental clinics around the state of Kelantan. Besides those forty samples, another three samples were also collected and were used as pilot samples. These collected teeth associated with the purpose of the treatment and prevention of disease and not for this research study purpose. The teeth that included were all intact crown with root structure, either right or left maxillary first molars were taken as a sample. Any samples with root caries or root canal treated teeth were excluded as they might affect the outcome of the results. After extraction, the teeth collected were stored

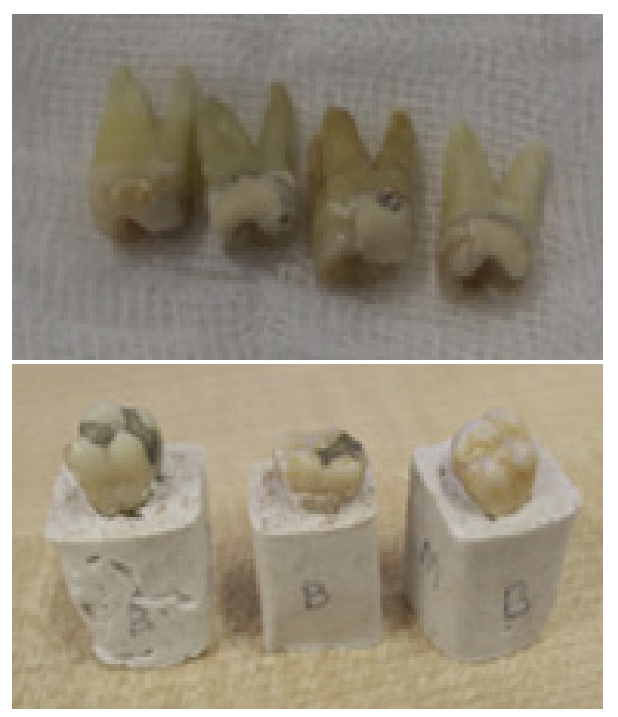

Figure 1. Upper: Samples that had been cleaned, disinfected and autoclaved. GIC restoration was placed on the tooth with gross caries on enamel; Lower; The teeth that had been mounted into a block made of Plaster of Paris with sawdust, then labelled to ease the identification process. 


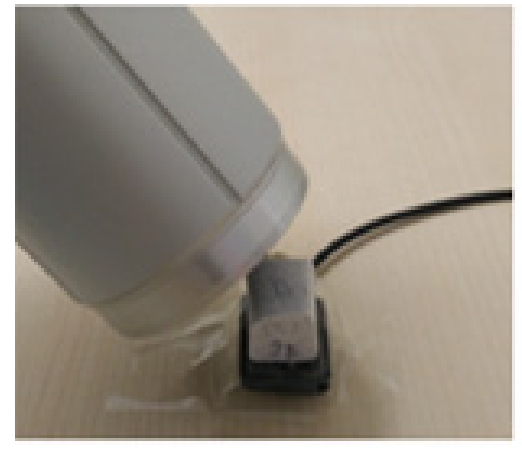

A

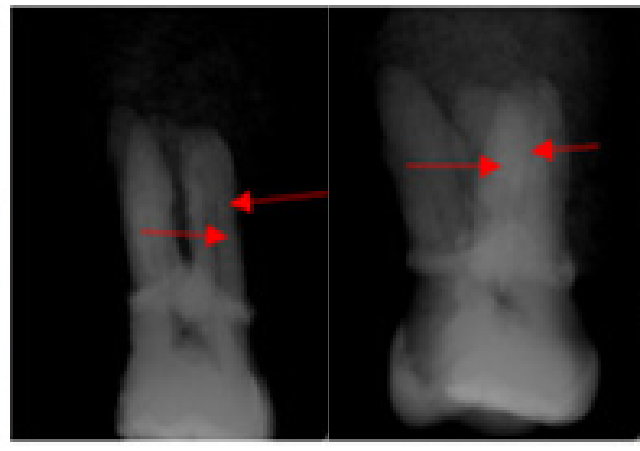

B

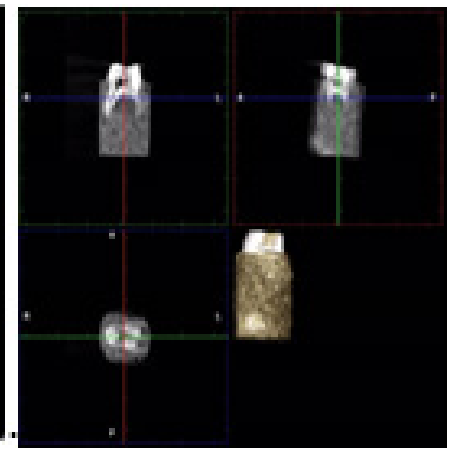

C

Figure 2: (a) Mesial SLOB PA X-ray technique was done on each tooth by tilting the tube mesially, (b) showed the presence of secondary MB root canal detected (red arrows). The secondary MB root canal could be seen well in the Mesial SLOB technique as compared to Buccal view, (c) The CBCT images that were produced into three different planes which were sagittal, axial, and coronal views.

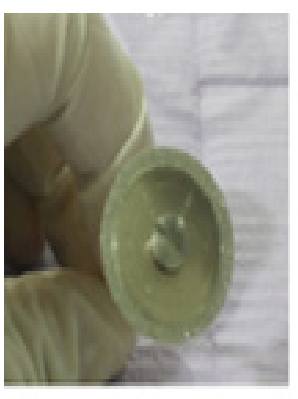

A

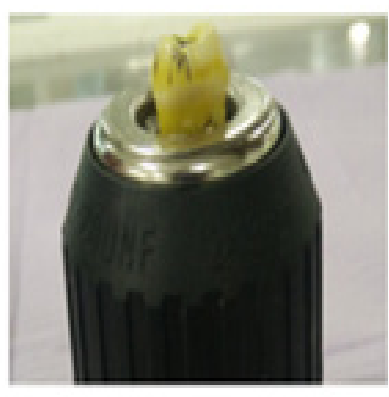

B

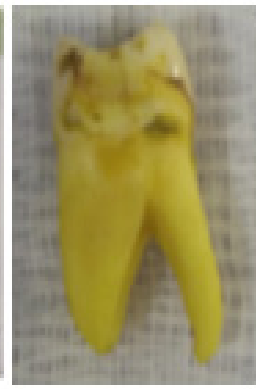

C

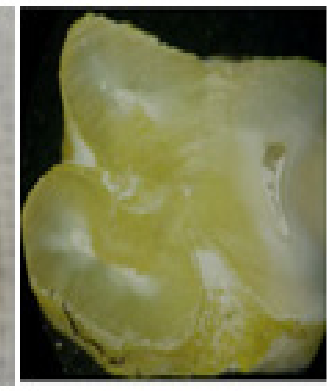

D

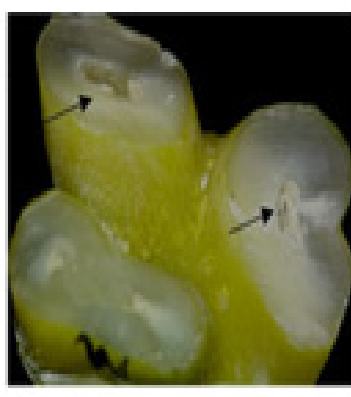

$E$

Figure 3. (a) Diamond disk bur was used for sectioning the samples, (b). The tooth was held during the sectioning process, (c) Tooth that has been immersed in an iodine solution for one minute and then dried before sectioning process, (d) Tooth sample that showed the presence of Secondary MB Root Canal at $3 \mathrm{~mm}$ after CEJ level. The arrows showed the first and second MB canal. (e) Sectioning was done at $3 \mathrm{~mm}$ before the apex of the tooth. The arrows indicated the presence of the first and second MB canals.

in the Dettol ${ }^{\circledR}$ antiseptic solution. The teeth were then cleaned by removing the debris using a supersonic scaler. After that, all teeth that had gross enamel and dentine caries were restored using Glass lonomer Cement $(\mathrm{GIC})$ to restore the tooth morphology for easier identification process (Fig. 1) during radiographic tests. The tooth blocks were made of the composition of Plaster of Paris and sawdust to increase the contrast during the radiographic evaluation process.

Digital periapical radiograph at imaging room Kulliyyah of Dentistry is used in this research. The first setup consisted of digital sensor that was placed directly under the $\mathrm{x}$-ray tube. The $x$-ray sensor was held by plasticine to avoid the sensor slipped. The $x$-ray sensor and $x$-ray tube were placed in the same position for every PA radiograph evaluation for each tooth. The blocks were placed directly onto the sensor in a Buccal-Lingual orientation, and the buccal side of the tooth was facing the tube. Two views were taken for every PA evaluation, which was buccal view and mesial SLOB (Side Lingual Opposite Buccal Technique). For every mesial view, the tube was tilted mesially of the mesial side of each tooth (Fig. 2a). The images from both buccal and mesial SLOB technique were then evaluated and documented (Fig. 2b). The presence or absence of secondary $M B$ root canal was noted as to compare in the $\mathrm{CBCT}$ radiograph and clinical sectioning.

Each tooth block then was incorporated into a piece of polystyrene block that a hole was made on top of the polystyrene to hold the block. The polystyrene also functioned to elevate the tooth blocks. The polystyrene was placed on top of the chin rest of the CBCT machine. The machine was then adjusted to make sure that the tooth was in the middle position of the mesial crown. A preview picture was taken to confirm the position of the sample. The exposure time was minimal, with 
minimal exposure radiation which was $9 \mathrm{~mA}$. The CBCT evaluations were operated by the students with the supervision from a radiographer in duty during the evaluation process. The final image was manipulated using every different cross section planes to evaluate the root and the presence of the secondary MB root canal (Fig. 2c). The results were evaluated by the students that later were checked by the endodontist. The inter-reliability tests were done between the endodontist and the students for every radiographic evaluation of $\mathrm{PA}$ and $\mathrm{CBCT}$ of every sample, including the pilot study test.

Each tooth was then sectioned by using diamond disk bur and the holder that functioned to hold the tooth (Fig. $3 a$ \& $3 b$ ). The samples were then removed from the blocks and cleaned. The blocks were cut by using stone trimmer in the laboratory. Every tooth was then cleaned again, and remnants of the Plaster of Paris were removed. Each sample was then placed into the iodine solution for one minute at room temperature, and dried before the clinical sectioning was done (Fig. $3 c)$.

Every tooth was sectioned at the cementoenamel junction (CEJ), and then $3 \mathrm{~mm}$ after the CEJ, and $3 \mathrm{~mm}$ coronal to the apex of the roots. All of the roots that were sectioned were then placed in their consecutive sample container that was initially labelled. The roots were then analyzed under the dental surgical microscope (Omni Pico () ). The number of the roots present were then determined from the evaluation through a microscope (Fig. 3d \& 3e). The processes were then repeated for all forty samples.

\section{RESULTS}

All 40 samples were evaluated using PA, CBCT, and Clinical Sectioning methods. Periapical Xray (PA) 40 samples, Cone Beam Computed Tomography (CBCT) 40 samples, and Clinical Sectioning (gold std) 40 samples.

Data analysis in Table 1, PA radiograph successfully identified eight samples presented with $M B 2$, while the $C B C T$ radiograph evaluation found 24 samples were presented with MB2 canal, and their percentage was $20 \%$ and $62.5 \%$ respectively. The sensitivity of the tests, when compared to the Gold Standard, was $27.6 \%$ and $86.2 \%$ for both PA and CBCT respectively. The $p$-value for the CBCT test compared to CS was 0.00 ; meanwhile, the $\mathrm{p}$-value for the PA test was 0.08 . This result proved that the test from $\mathrm{CBCT}$ as compared to the gold standard method was statistically more significant than PA radiograph $(p<0.05)$.

Specificity and sensitivity of the test between PA radiograph and CS were further evaluated using Kappa statistical analysis and the yields from the result were assessed by the positive predictive value (PPV) and negative predictive value (NPV) from the Chi-Square test. From Table 2, Kappa value correlation was only 0.173 between these tests. Besides that, it also showed the reduced specificity of PA test since the NPV of the PA radiograph was only $34.4 \%$.

Data analysis comparing the sensitivity and specificity between the CBCT and CS was done by using Kappa and the yields of the result (PPV and NPV) from the Chi-Square test were tabulated in Table 3. Kappa value between the CBCT and CS was 0.775 , and their NPV was $73.3 \%$.

Table 1. Sensitivity differences between PA and CBCT radiograph in evaluating the presence of $M B 2$ and their respective $\mathrm{p}$-value

\begin{tabular}{llll}
\hline & PA & CBCT & CS - Gold standard \\
\hline Has MB2 & 8 & 25 & 29 \\
No MB2 & 32 & 15 & 11 \\
p value & 0.08 & 0.00 & \\
\hline
\end{tabular}

Table 2. Correlation between the PA test and gold standard with their respective Kappa value

\begin{tabular}{llll}
\hline & PA (in \%) & CS-Gold standard (in \%) & Kappa \\
\hline \multirow{2}{*}{ Has MB2 } & $8(27.6 \%)$ & $29(72.5 \%)$ & 0.173 \\
& PPV: $100 \%$ & PPV: $100 \%$ & \\
\multirow{2}{*}{ No MB2 } & $32(72.4 \%)$ & $11(27.5 \%)$ & \\
& NPV: $34.4 \%$ & NPV: $100 \%$ & \\
\hline
\end{tabular}

Table 3. Correlation between the CBCT radiograph and gold standard with their respective Kappa value

\begin{tabular}{llll}
\hline & CBCT (in \%) & CS-Gold standard (in \%) & Kappa \\
\hline \multirow{2}{*}{ Has MB2 } & $25(86.2 \%)$ & $29(72.5 \%)$ & 0.775 \\
& PPV: $100 \%$ & PPV: $100 \%$ & \\
\multirow{2}{*}{ No MB2 } & $15(13.8 \%)$ & $11(27.5 \%)$ & \\
& NPV: $73.3 \%$ & NPV: $100 \%$ & \\
\hline
\end{tabular}




\section{DISCUSSION}

Radiographic imaging can be considered as an essential investigation, specifically in endodontic cases as it is an important method in diagnosis, treatment planning and also in for the evaluation after the endodontic treatment. According to the research conducted by Patel et al. ${ }^{1}$ in 2007 that was agreed by American Association of Endodontist in 2011, the most outstanding advantage of CBCT is that it produces images in $3 \mathrm{D}$ views, the images will be presented into three planes it's sagittal, axial and coronal, the images also can be zoomed without any distortion. Causative of these factors, this study was aimed to prove that $C B C T$ is more accurate as compared to PA radiograph in identifying the presence of Secondary $M B$ root canal in maxillary first molars.

From the research, found that the percentage of secondary $M B$ root canal present by the clinical sectioning evaluation it's $72.5 \%$, PA $20 \%$, and CBCT $62.5 \%$. Based on the data from the research found that the percentage of the secondary $M B$ root canal evaluation by the $C B C T$ was slightly lower than the literature, which has suggested that the prevalence of Secondary MB root canal in Malay population was $68 \% .{ }^{5}$ However, the smaller sample size could be the main reason accounted for this difference.

Another relevant study was done based on the population by Abd Latib et al. ${ }^{6}$ in 2015 found that there were 15 canals of Secondary MB root canal detected among the Malay population, while among Chinese population there were five canals found and none was found among Hindi population. ${ }^{6}$ Besides that, another study in the prevalence of Secondary $M B$ root canal in maxillary first molars by Peiris et al. ${ }^{7}$ in 2012 among Sri Lankan population found that $77.1 \%$ of the samples presented with the Secondary MB root canal. ${ }^{7}$ Another study conducted among the Indonesian population by Peeters et al. ${ }^{8}$ found that $68.5 \%$ of the total extracted 308 samples has the Secondary MB root canal. ${ }^{8}$ These percentages of the presence of Secondary MB root canal among the Asian showed significant value as the prevalence among these populations were nearly the same.

In addition to the prevalence studies, the validity of the study also has been confirmed by evaluating the sensitivity and specificity of the screening methods. The results from these screening methods were compared to the clinical sectioning (Gold Standard) findings. However, in terms of specificity, both CBCT and PA radiograph most high specificity in detecting the presence of Secondary MB root canal. This was confirmed by the ability of both methods, as well as the gold standard to successfully detect that 11 true negative samples which absent of Secondary MB root canal.

Although the test by CBCT was not $100 \%$ as accurate as the gold standard, this result has proved that $C B C T$ was highly sensitive if compared to $\mathrm{PA}$ radiograph in detecting the presence of Secondary $M B$ root canal. This finding was due to the higher percentage of true positive results compared to the PA test that was illustrated by the sensitivity of the tests. Besides that, Kappa value also higher in $C B C T$ those values showed the high correlation and reliability of the CBCT more than PA radiograph when compared to the gold standard method. Therefore, the accuracy of $C B C T$ was confirmed as higher if compared to the $\mathrm{PA}$ radiograph as the percentage difference between CBCT and gold standard was not too significant.

Apart from the sensitivity and specificity of the test, the predictive value also was evaluated by the statistics. The positive predictive value (PPV) and negative predictive value (NPV) were evaluated as yields of the screening test. Any evaluation test with high PPV and NPV confirmed that the test was an ideal test. In this study, both PA and CBCT evaluation test had produced a very high PPV; it shows in the evaluation of the present of Secondary MB root canal, PA radiograph accounted a lower NPV than CBCT.

By all these results, they demonstrated that $\mathrm{CBCT}$ has the superiority in the diagnosing the presence of the Secondary MB root canal compared to $\mathrm{PA}$ radiograph, this is similar to the research by Blattner et al. in 2010. ${ }^{4}$ On the other hand, a study was done by Michetti et al. ${ }^{9}$ in 2010 found that the CBCT has high validation as a method to explore the root morphology, when compared to the histological sectioning. They studied on the ability of the CBCT in reconstruction the root morphology, and their results proved the strong correlations between the CBCT and sectioning with the standard deviation value was $0.163 .{ }^{9}$ 
The differences in the percentage of the correlation between the CBCT and Clinical Sectioning could be credited to the different number of samples, and the origin of the population itself. This study has been conducted without any consent taken from the patient as the tooth collected was anonymous and the races or origin of the tooth could not be determined from the samples collection.

Therefore, further improvement in this study should consider the samples collected from a specific population so that the prevalence and the incidence that certain population could be determined. Another possible factor that could be considered was the gender of the patient from which the samples had been collected because gender predilection could be among the factors. However, there was no definite result or studies conducted. Besides that, an increment in the sample size also should be considered so that higher positive correlation between the CBCT and the gold standard could be anticipated. This was because smaller range of the sample size would limit the accountancy of the presence or absence of the Secondary MB root canal in the overall number of the population's prevalence.

Apart from all these factors, this study also should consider being conducted on the real patient with the need of endodontic therapy so that a practical application of the research could be optimized. Instead of having the clinical sectioning as the screening test, the usage of the clinical surgical microscope which was currently widely used in endodontic treatment could be used to evaluate the presence of Secondary MB root canal by using canal patency identification technique.

\section{CONCLUSION}

CBCT was proven to be a reliable method to detect the presence of secondary $M B$ root canals due to its accuracy as high as the clinical sectioning compared to the PA radiograph.

\section{REFERENCES}

1. Patel S, Dawood A, Ford TP, Whaites E. The potential applications of cone beam computed tomography in the management of endodontic problems. Int Endod J 2007;40(10):818-30. DOI: 10.1111/j.1365-2591.2007.01299.x.

2. Scarfe WC, Levin MD, Gane D, Farman AG. Use of Cone Beam Computed Tomography in Endodontics. Int J Dent 2009: 634567. DOI: 10.1155/2009/634567.

3. Somma F, Leoni D, Plotino G, Grande NM, Plasschaert A. Root canal morphology of the mesiobuccal root of maxillary first molars: a micro-computed tomographic analysis. Int Endod J 2009;42(2):165-74. DOI: 10.1111/j.1365-2591.2008.01472.x.

4. Blattner TC, George N, Lee CC, Kumar V, Yelton CD. Efficacy of Cone-Beam Computed Tomography as a modality to accurately identify the presence of second mesiobuccal canals in maxillary first and second molars: A pilot study. J Endod 2010;36(5):867-70. DOI: 10.1016/j.joen.2009.12.023.

5. Majeed A, Raad K, Mustafa NS. The accuracy difference between surgical microscope and CBCT to find MB2 canal in mesiobuccal root of maxillary permanent first molar in Malay population. $39^{\text {th }}$ Annual Conference of the European Prosthodontic Association; Sep 3-5, 2015; Prague, Czech Republic: European Prosthodontic Association; 2015.

6. Abd Latib AH, Nordin NF, Alkadhim AH. CBCT Diagnostic Application in Detection of Mesiobuccal Canal in Mandibular Molars: A Descriptive Study. J Dent Oral Health 2015;1(1):1-3.

7. Peiris R. Mesiobuccal root canal morphology of the permanent maxillary first molar in A Sri Lankan population. Malay Dent J 2012;34(2):36-44.

8. Peeters HH, Suardita K, Setijanto D. Prevalence of a second canal in the mesiobuccal root of permanent maxillary first molars from an Indonesian population. J Oral Sci Dec 2011;53(4):489-94.

9. Michetti J, Maret D, Mallet JP, Diemer F. Validation of Cone Beam Computed Tomography as a tool to explore root canal anatomy. J Endod 2010;36(7):1187-90. DOI: 10.1016/j.joen.2010.03.029. 